\title{
Rôle des Comités d'éthique dans la formation médicale
}

\author{
Gry LLORCA*
}

\begin{abstract}
Résumé Contexte: L'éthique occupe une place de plus en plus importante dans le raisonnement et, particulièrement, la décision médicale. Son enseignement soulève des difficultés car il comporte une partie fondamentale (discursive) et une partie appliquée (contextuelle). Il met en jeu une approche obligatoirement pluridisciplinaire, il nécessite la coopération de méthodes de formation centrées sur l'apprentissage de l'analyse documentaire et décisionnelle contextuelle ainsi que sur l'apprentissage de l'élaboration d'avis collectifs basés sur le principe de l'accord librement consenti. Du fait de ces difficultés et de la rigidité des programmes de formation, cet enseignement est encore trop peu développé en France. But: Les Comités locaux d'éthiques, par leur vocation naturelle et leur potentiel pluridisciplinaire, peuvent apporter une contribution notable à l'enseignement de l'éthique, particulièrement dans son domaine appliqué et clinique. Méthode : L'expérience du Comité d'éthique du Centre hospitalo-universitaire de Lyon est rapportée à propos de 30 stagiaires accueillis en 6 ans. L'évaluation en termes d'opinion des stagiaires qui ont participé à cette expérience a été très favorable. Résultats : Cette expérience est susceptible d'enrichir les formations fondamentales à l'éthique puisque les stagiaires sont confrontés à l'étude de cas réels, quills ont accès aux archives des situations traitées dans le passé par les Comités et sont confrontés aux opinions des différents membres permanents. Les Comités d'éthiques locaux sont suffisamment nombreux pour que cette possibilité de formation soit proposée de manière plus systématique. Elle nécessite cependant la disponibilité de tuteurs compétents et expérimentés pour encadrer valablement les stagiaires et leur permettre de progresser.
\end{abstract}

Mots clés Ethique ; bioéthique ; comité d'éthique ; enseignement médical.

Summary Context: Ethics holds an important place in reasoning and particularly in medical decision making. Teaching ethics raises difficulties because it involves fundamental and practical parts, it also needs a multidisciplinary approach, co-operative methods of education centred on moral reasoning and judgement making based on the rules of mutual agreement. Because of these difficulties and the rigidity of medical programs, teaching ethics is yet little developed in France. Object: To assess if local ethics committees, with their natural vocation and multidisciplinary approach potential, can notably contribute to this teaching. Method: The experience of the local ethic committee of Lyon with 30 students during 6 years is described. Evaluation, based on the opinion of the students was very favourable. Results: This kind of experience can contribute significantly to the teaching of ethics as the students are exposed to real cases, they can have access to the large documentation accumulated by the committee in the past and they are confronted with the different opinions of permanent committee members. The local ethics committee are in sufficient number to propose this formation in a more systematic way. However, a great availability of competent tutors is needed to assist the students and to give them an opportunity to learn.

Key words Ethics; bioethics; ethics committee; medical education.

Pédagogie Médicale $2001 ; 2: 157-162$

Conférence présentée le 4 avril 2001, lors des XIV^s Journées Universitaires Francophones de Pédagogie Médicale de la CIDME - Ouagadougou (Burkina Faso)

* Président du Comité d'Ehique du CHU de Lyon, Laboratoire de Thérapeutique, 8, avenue Rockefeller 69373 Lyon Cedex 08 France - Courrier électronique : Ilorca@cismsun.univ-lyon1.fr 


\section{Concepts et Innovations}

\section{Introduction}

Au-delà d'une mode, l'éthique représente une dimension fondamentale de la décision médicale. La prise de conscience de cette importance est récente (quelques décennies) et son caractère pluridisciplinaire n'en facilite pas l'enseignement. Si de nombreuses tentatives ont été tentées ${ }^{1}$, l'éthique a encore de la peine à figurer dans les programmes universitaires, tout du moins en France. Depuis les années 80, l'individualisation de la bioéthique, l'activité des Comités d'éthiques locaux et la progression de la réflexion pédagogique permettent de distinguer plus clairement les possibilités concrètement utilisables. L'éthique, pratique discursive et discours pratique selon la formule de G. Hottois ${ }^{2}$, comporte, à l'évidence, un domaine fondamental théorique et un domaine pratique, particulièrement en médecine. L'enseignement des bases fondamentales est relativement facile à assurer à l'occasion de formations traditionnelles. Le domaine pratique soulève plus de difficulté par la particularité de la démarche qu'il nécessite. Les Comités d'éthiques locaux peuvent jouer un rôle manifeste dans cette approche puisqu'ils sont plus ancrés sur la réalité des problèmes cliniques et qu'ils regroupent des compétences multiples susceptibles d'aider ainsi l'apprentissage du raisonnement analogique et de la démarche pluridisciplinaire.

\section{De quoi s'agit-il?}

Vouloir définir l'éthique représente la première difficulté de son enseignement. De nombreuses et belles définitions en ont été données, particulièrement par Jean Bernard ${ }^{3}$. Pour demeurer pratique, nous considérons l'éthique comme " la discipline du jugement de valeur dans la décision humaine ». En effet, comme l'a indiqué Emmanuel Levinas, l'éthique fonde l'ontologie et doit être une logique tournée vers le réel. Si elle peut être considérée comme l'art du meilleur usage de soi, elle est aussi régulatrice de la communication humaine en procédant de l'équilibre réfléchi de Rawls ${ }^{4}$ et de l'accord librement consenti d'Engelhardt ${ }^{5}$. Source déontologique comme l'a montré Emmanuel Kant, elle la dépasse et traite, non seulement de l'humain, mais de tous les êtres sentants ${ }^{6}$. Elle est devenue biocentrée ${ }^{7}$ et, en fin de compte, devant les nouvelles questions soulevées par les immenses progrès de la médecine conduisant à une thérapeutique efficace, elle ne s'adapte pas mais permet de réinterpréter et d'ac- tualiser les valeurs fondamentales au fur et à mesure des nouvelles questions que la science pose à l'humanitér. Elle est contenue dans la définition poétique que Pierre Reverdy nous donne dans "En vrac": "l'éthique c'est l'esthétique du dedans », complétée par cette phrase redoutable d'Albert Camus dans « La peste ": "il n'a jamais tué une mouche... la mouche qu'il aurait pu tuer est allée porter la peste. »

\section{Faut-il l'enseigner?}

On est surpris, lors d'études des attentes et besoins de formation exprimés par les praticiens ${ }^{9}$, de ne pas voir figurer l'éthique comme besoin spontanément exprimé. Il s'agit, en fait, plus d'un " effet scotome " classique dans ce genre d'enquêtes car, lors d'interrogations directes sur le sujet, une quasi unanimité se dégage pour constater le caractère quotidien, en pratique de soins, des problèmes d'ordre éthique, et pour confirmer l'utilité de l'étude de cette dimension. Au plan décisionnel, l'éthique apparaît comme l'un de 6 facteurs cardinaux qui doivent guider le raisonnement médical et la décision de prescription (figure 1). Elle apparaît, même, comme le seul facteur stable, puisque les dimensions descriptives sont éminemment variables par nature et, parmi les facteurs que nous désignons comme normatifs, les données de la science doivent être sans cesse actualisées dans le cadre de "l'evidence based medicine " et le facteur légal leur est logiquement et directement lié. Devant les avancées actuelles et à venir de la médecine, on peut valablement penser que cette dimension éthique, par son universalité, deviendra le facteur décisif là où aucune réponse satisfaisante ne peut être valablement apportée par les autres dimensions. La relation médecin-malade devient de plus en plus exigeante avec l'évolution des mœurs et des consciences ainsi elle évolue vers un véritable contrat "médecin-bénéficiairecollectivité ». Cette relation ne peut être sérieusement établie que sur des bases éthiques solides faisant de cette discipline une véritable discipline du savoir-être en médecine. L'éthique doit, à l'évidence, être enseignée.

\section{Intentions pédagogiques et contenu}

Si l'éthique doit être enseignée, comment peut-elle l'être ? La réponse à cette question est contenue dans la définition des intentions pédagogiques ${ }^{10}$. Pour qu'un tel enseignement soit utile et riche, il doit, avant tout, 


\section{Figure 1 : Facteurs de la décision médicale}

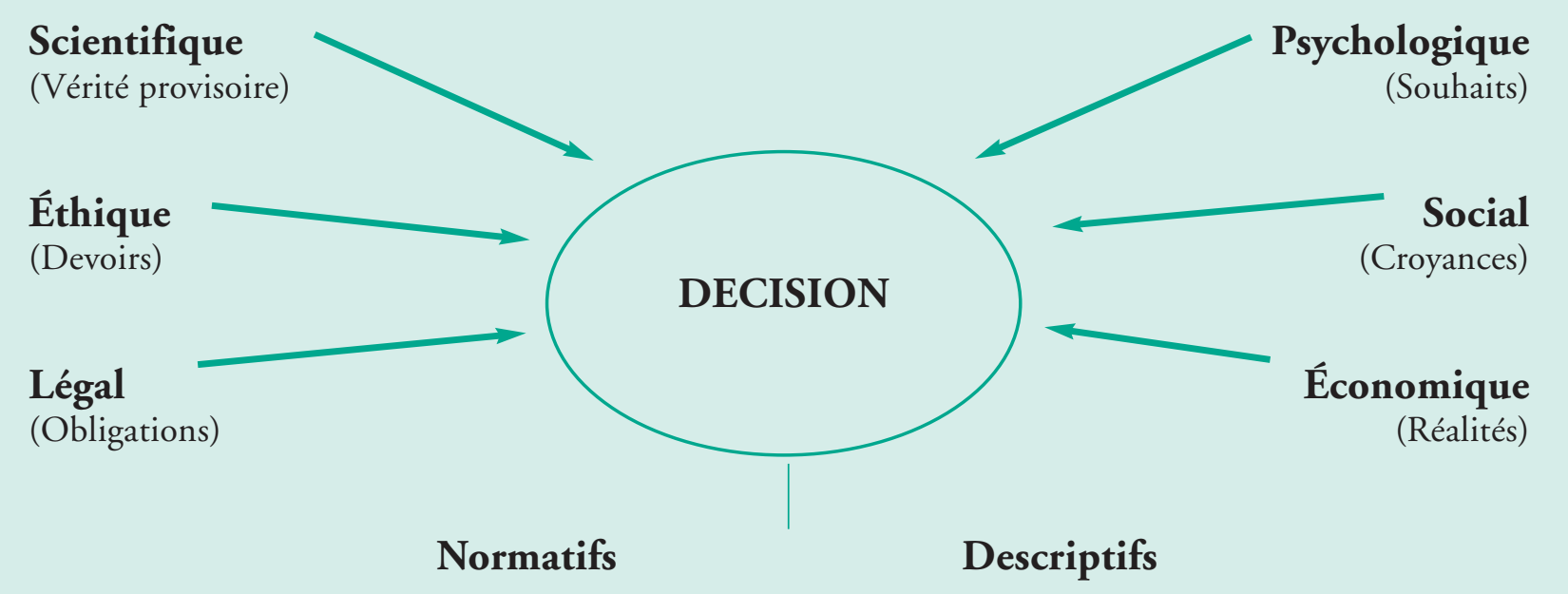

mettre l'accent sur les modalités de raisonnement qui permettent d'aboutir à l'avis éthique documenté (qu'il soit d'ordre universel ou analogique), avis qui guidera la décision et ses conséquences. Cette dynamique, schématisée (figure 2), permet d'identifier l'importance de l'apprentissage des prémices fondamentales, des modalités analytiques et documentaires ainsi que de la confrontation groupale des constructions élaborées. Une telle diversité ne peut valablement être couverte par une méthode pédagogique unique, si riche soit-elle. L'enseignement de l'éthique nous semble, à cet égard, l'un des meilleurs exemples de pédagogie obligatoirement multimodale ${ }^{11}$.

Le contenu correspondant aux objectifs généraux de cet enseignement a été maintenant bien identifié ${ }^{12}$. Il couvre les deux domaines admis de l'éthique : domaine discursif correspondant aux bases fondamentales et domaine appliqué correspondant aux bonnes conduites :

\section{Domaines de l'éthique}

\begin{tabular}{|l|l|}
\hline Ethique discursive & Ethique appliquée \\
\hline - Philosophie Morale & • Bioéthique \\
- Méta-éthique & (éthique médicale \\
- Courants modernes & et éco-éthique) \\
(principisme, éthique & - Ethique profession- \\
procédurale, & nelle (des affaires, \\
contextualisme...) & de l'ingénierie, des \\
& communications...)
\end{tabular}

L'enseignement du domaine discursif peut être réduit en médecine, mais doit faire comprendre les trois attitudes habituellement rencontrées en matière de décision : attitude "naturaliste " privilégiant le respect des choses naturelles, attitude "progressiste " privilégiant le progrès scientifique même contre nature, et attitude "prudentialiste » tentant d'encadrer les décisions de critères normatifs pour respecter nature et progrès. L'enseignement de ce domaine doit aussi comporter l'étude des courants modernes : courant principiste de Beauchamp et Childress ${ }^{13}$, éthique procédurale d'Engelhardt ${ }^{5}$, courant contextualiste de Jonsen et Toulmin ${ }^{14}$. L'ensemble de cet enseignement permet la réflexion téléologique, l'étude des fondements de la critique sociale ${ }^{15}$ et de l'agir communicationnel ${ }^{16}$ pour aboutir à la finalité de la médecine, " art de cultiver l'autonomie des Hommes en prenant soin de leur corps » selon la formule de Jean-François Malherbe ${ }^{17}$.

La bioéthique représente le domaine appliqué majeur qui doit être enseigné en médecine, particulièrement dans le cadre des trois champs suivants :

1 - L'éthique clinique : qui correspond au champ des bonnes conduites et pratiques de soins. C'est le domaine du consentement, de la confidentialité, de l'information, du refus de soin, des notions de responsabilité, de bénéfice, de maléfice, de qualité de vie, etc. 2 - La régulation de la recherche clinique : qui correspond aux normes de bonnes pratiques de recherche, qu'elles soient ou non encadrées par une loi comme c'est le cas en France. 


\section{Concepts et Innovations}

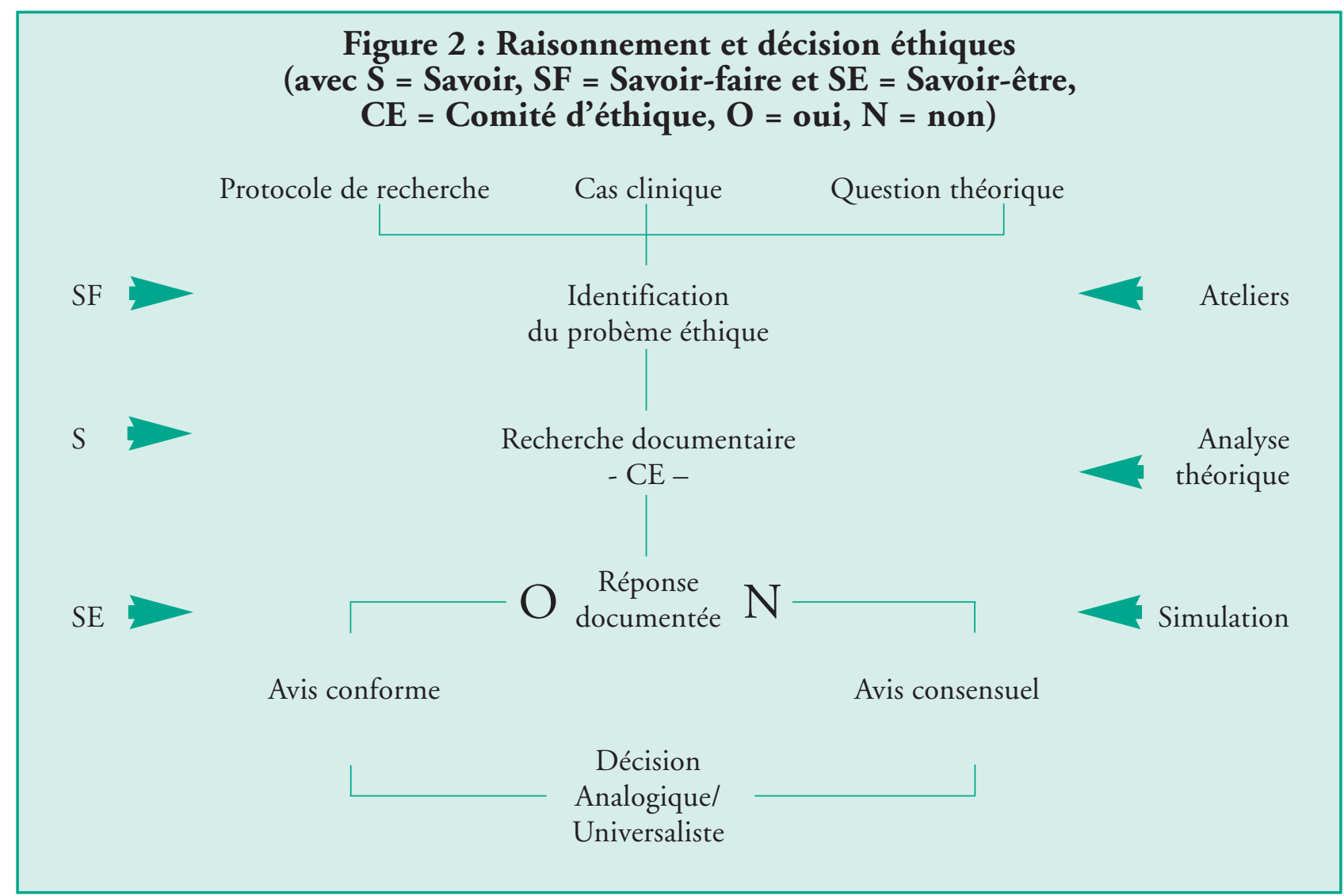

3 - L'éthique des techniques : elle concerne les problèmes posés par la procréation (procréation médicalement assistée, diagnostic prénatal, statut de l'embryon...), par le patrimoine génétique (conseil génétique, médecine prédictive, thérapie génique...), par l'intervention sur le corps humain (transplantations d'organes ou de tissus, transsexualisme, médecine esthétique, chirurgie plastique...), par l'intervention sur la personnalité (psychochirurgie, psychopharmacologie, psychothérapies...), par la fin de vie et la mort (euthanasie, acharnement thérapeutique, testament biologique...), enfin, par l'environnement (éco-éthique appliquée à la médecine).

\section{Place des Comités dans les possibilités actuelles et expérience de Lyon}

Actuellement, en France, les étudiants en médecine en formation initiale peuvent avoir une sensibilisation théorique à l'éthique par l'intermédiaire des enseignements dispensés dans le cadre des sciences humaines et sociales, en début de cursus. Par la suite, les problèmes spécifiques liés à certaines pathologies ou thérapeutiques peuvent être envisagés (exemple des items éthiques du nouveau cursus de second cycle des études médicales françaises). Une formation plus fondamentale peut être acquise par l'intermédiaire de certificats ou diplômes spécifiques facultatifs, formations dispensées en faculté de médecine ou à l'extérieur de ce contexte. La formation pratique est essentiellement une formation " sur le tas ", fonction des stages pratiques, de leur nature et des compétences de leurs responsables. Par la suite, en formation continue, des ateliers d'éthique centrés sur des problématiques pratiques et travaillant sur un mode casuistique représentent certainement la modalité la mieux adaptée mais sont encore trop peu développés et demandent une animation pluridisciplinaire délicate à rassembler.

Les Comités d'éthiques locaux nés en France à la suite de la Déclaration dite de Tokyo de la 29ª Assemblée médicale mondiale de 1975, peuvent représenter un lieu privilégié de formation pratique complémentaire, particulièrement lorsqu'ils sont rattachés à un centre hospitalo-universitaire (plus d'une quinzaine en France). A Lyon, le Comité d'Ethique, rattaché aux 
Hospices Civils et à l'Université Claude Bernard, a été créé en 1981. Depuis 1995 il accueille des stagiaires par petits groupes dans le cadre d'une unité de valeurs de $3^{\mathrm{e}}$ cycle d'études médicales. Ces stagiaires deviennent membres provisoires du Comité pour une durée d'au moins 6 mois. Chaque stagiaire est encadré par un tuteur, membre titulaire du Comité. Chaque stagiaire doit respecter le règlement interne du Comité (obligation de réserve particulièrement), doit étudier les dossiers dont le Comité est saisi, doit participer aux réunions plénières et aux débats, doit réaliser une communication devant le Comité pendant son stage. Chaque stagiaire a accès à la documentation du Comité (dont les actuels 1500 dossiers traités et documentés pendant ces 20 dernières années).

L'objectif général de cette formation rejoint l'intention pédagogique énoncée : être capable d'identifier un problème éthique et être capable de documenter un avis pour aboutir à un accord librement consenti. Les objectifs opérationnels et spécifiques sont essentiellement centrés sur l'éthique appliquée et, particulièrement, l'éthique clinique à l'occasion des cas soumis au Comité et étudiés pendant la durée du stage ou de cas extraits des annales du Comité ou de l'expérience du stagiaire. Du fait du niveau d'études des stagiaires, l'évaluation a été délibérément formative. Le tuteur a veillé, en s'adaptant au niveau de connaissances en éthique fondamentale du stagiaire, à la réalisation des objectifs opérationnels et spécifiques fixés individuellement. Dans certains cas, à la demande du stagiaire, des objectifs spécifiques concernant l'éthique des techniques ont également été déterminés et approfondis avec les experts du domaine considéré. Une enquête d'opinion sur l'ensemble des stagiaires concernés objective un indice de satisfaction satisfaisant : l'assiduité de ces stagiaires $(\mathrm{N}=30)$ a toujours été excellente, leur satisfaction $(8,7 \pm 0,8 / 10)$, leur perçu d'intérêt $(9,0 \pm 1,1 / 10)$ ou d'utilité $(8,3 \pm 0,9 / 10)$ sont également satisfaisants. Leur motivation à poursuivre est établie pour $87 \%$ d'entre eux ( 2 d'entre eux ont même rédigé une thèse concernant l'éthique en médecine).

Ainsi, par leur vocation naturelle et leur potentiel ${ }^{18}$, les Comités d'éthique représentent un lieu privilégié pour l'enseignement pratique de l'éthique, non seulement des personnes qui les saisissent, mais aussi de stagiaires étudiants en médecine, médecins diplômés ou personnels paramédicaux. Ces Comités permettent l'étude de dossiers réels et l'approfondissement, à cette occasion, du raisonnement éthique par l'approche des fondements, de l'analyse problématique, d'une documentation casuistique particulièrement riche et de la réflexion en groupes pluridisciplinaires qui existent de fait, réalisant une pédagogie de type collaborative ${ }^{1}$ et facilitent le recours aux experts concernés lorsque les expériences et compétences des tuteurs sont insuffisantes. La participation de ces stagiaires aux débats est très souvent également enrichissante pour ces Comités, leur apportant la sensibilité de jeunes professionnels de Santé en début de carrière. Les possibilités de méthodes pédagogiques d'analyse théorique, d'ateliers de travail et de simulation sont heureusement réunies au sein de telles structures (figure 2). Chaque tuteur peut enfin, s'il le souhaite, élaborer avec le stagiaire concerné une évaluation formative critériée, en fonction des thématiques étudiées.

Les difficultés de cet enseignement sont représentées par la nécessité de disposer d'un Comité d'Ethique de structure pluridisciplinaire et de tuteurs expérimentés et volontaires. En effet, ce tutorat demande une disponibilité particulière pour aider les stagiaires à rassembler la documentation nécessaire, pour suivre leur progression individuelle dans le cadre des intentions et objectifs pédagogiques définis et traiter les nombreuses questions et problèmes qu'ils soulèvent immanquablement au fil des cas étudiés ou à la suite des positions adoptées.

\section{Conclusion}

Le bilan de six années d'accueil de stagiaires au Comité d'Ethique du CHU de Lyon est positif en termes de satisfaction des stagiaires et des membres du Comité. Il met l'accent sur les possibilités naturelles de ces Comités en matière d'enseignement de l'éthique, enseignement indispensable mais délicat du fait de sa nécessaire pluridisciplinarité et de son manque de "discipline naturelle d'accueil ». Ces possibilités s'expriment pleinement dans le cadre de l'apprentissage du raisonnement et de la décision en matière d'éthique, apprentissage qui doit être dynamique et centré sur le réel. 


\section{Concepts et Innovations}

\section{Références}

1. Marcoux H. et Patenaude J. L'éthique et la formation médicale. Où en sommes-nous? Où allonsnous? Pédagogie Médicale 2000 ; 1 : 23-30.

2. Hottois G. Le paradigme Bioéthique. Une éthique pour la technoscience. Bruxelles: Editions DeBoeck, 1990.

3. Bernard J. La bioéthique. Paris : Editions Flammarion (dominos), n 15, 1994.

4. Rawls J. Théorie de la justice. Paris : Editions Le Seuil, 1987.

5. Engelhardt T. : The Fondations of Bioethics. Oxford: Oxford University Press Edition, 1986.

6. Singer P. : Practical Ethics. Cambridge : Cambridge University Press Edition, 1979.

7. Parizeau M.-H. : Les Fondements de la bioéthique. Bruxelles, Montréal : Editions DeBoeck/ERPI, 1992.

8. MacIntyre A. : Does applied ethics rest on a mistake? The Monist. 1984, 67: 498-513.

9. Bui-Xuan A.S. : Recueil des attentes et identification des besoins de formation des médecins généralistes du Rhône. Lyon: Thèse médecine, 2000.

10. Hameline D. : Les objectifs pédagogiques en formation initiale et en formation continue. Paris : Editions ESF, 1998.
11. Llorca $G$. : La formation médicale (aspects conceptuels). Lyon : Méditions, 1999.

12. Parizeau M.H. : Bioéthique. Ethique appliquée. In : Canto-Sperber M. : Dictionnaire d'éthique et de philosophie morale. Editions Presse Universitaire de France. Paris : ( $1^{\circ}$ édition 1996), $2^{\circ}$ édition 1997 : 155-160 et 534-540.

13. Beauchamp T. \& Childress J. : Principles of Biomedical Ethics. New York, Oxford: University Press Edition., (1ºdition 1979), $3^{\circ}$ edition, 1989.

14. Jonsen A. et Toulmin S. : The abuse of casuistry : A history of moral reasoning. Berkeley: University of California Press Edition, 1988.

15. Baynes K. : The Normative Grounds of Social Criticism. Kant, Rawls and Habermas. New York: State Univ. of New York Press, 1992.

16. Habermas J. : Théorie de l'agir communicationnel. 2t. Paris : Editions Fayard, 1987.

17. Malherbe J.F. : Pour une éthique de la médecine. Namur, Montréal : Editions catalyses/Artel-Fides, 1997.

18. Parizeau M.H. : Ethique et Hôpital : rôles et défis des comités d'éthique clinique. Québec: Editions Presses Universitaires Laval, 1995. 